Proceedings of the IV Brazilian Conference on Neural Networks - IV Congresso Brasileiro de Redes Neurais pp. 344-349, July 20-22, 1999 - ITA, São José dos Campos - SP - Brazil

\title{
A Neurocomputational Model for Autism
}

\author{
Luís Alfredo Vidal de Carvalho ${ }^{1}$, Nivea de Carvalho Ferreira ${ }^{2}$, Adriana Fiszman ${ }^{3}$ \\ ${ }^{1,2}$ COPPE/UFRJ - Program of Systems Engineering and Computer Science \\ PO. Box 68511 - Rio de Janeiro, 21945-970, RJ, Brazil \\ ${ }^{3}$ IPUB/UFRJ - Institute of Psychiatry \\ Av. Venceslau Bras, Rio de Janeiro, Brazil \\ E-mails: OKay@IamWaiting.com, niveacf@cos.ufrj.br, afiszman@ rio.com.br
}

\begin{abstract}
Autism is a mental disorder characterized by deficits in socialization, communication, and imagination. Along with the deficits, autistic children may have savant skills ("islets of ability") of unknown origin. The present article proposes a neurobiological model for the autism. A neural network capable of defining neural maps simulates the process of neurodevelopment. The computer simulations hint that brain regions responsible for the formation of higher level representations are impaired in autistic patients. The lack of this integrated representation of the world would result in the peculiar cognitive deficits of socialization, communication, and imagination and could also explain some "islets of abilities". The neuronal model is based on plausible biological findings and on recently developed cognitive theories of autism. Close relations are established between the computational properties of the neural network model and the cognitive theory of autism denominated "weak central coherence", bringing some insight to the understanding of the disorder.
\end{abstract}

\section{Introduction}

Autism is a psychiatric disorder that was first described by Leo Kanner in 1943. This researcher observed four fundamental characteristics in the disease: extreme autistic aloneness, failure to use language in a communicative fashion, obsessive desire for the maintenance of sameness, and a tendency to repeat the same action in a ritualized manner [14]. Concurrently, though independently, Hans Asperger also used the term autism to refer to the core features of the disorder [1].

The increased perception of infantile autism as a distinct nosological entity led to a great deal of research and clinical speculation and to the development of the first operationally precise diagnostic criteria in 1978 $[20,22]$. Almost at the same time, other authors [24] defined what they thought to be the core features of the disease, namely impairments in socialization, communication, and imagination. These features form a triad, since they co-occur and persist during the development of the child even though their outward manifestation is subject to change.
Along with the lack of gesture or verbal language, poor eye contact, echolalia, perseveration, and low generalization capability, some autistic patients (approximately one case in ten) display islets of exceptional abilities against a background of widespread cognitive impairment (so-called "savant skills"), such as calendar and prime number calculation, counting, memory, drawing, and music. The higher capability in performing these tasks is not learned but it just emerges spontaneously without explanation until now and it does appear to be specific. Other types of mental handicap or developmental disorder do not share this profile [9].

Mental retardation is one of the most strongly associated, but not the defining, features of autism. About three-quarters of the autistic population have IQs in the retarded range. Regardless of the low overall IQ, the intellectual abilities of the autistic children show marked variations, ups and downs commonly called "spiky profile", that have been intriguing the researchers until now.

Indeed, a neuronal model was proposed some years ago exploring the findings of increased or reduced neurons and synapses in autistic brains [2]. Computer simulations of the neural network there proposed have shown that good discrimination of stimuli is accomplished when there are too many neurons in one specific layer of the neural network, while generalization properties are obtained only when few neurons are present in the referred layer. The author associated these well-known results of the theory of artificial neural networks [21] with the deficits of concept acquisition and generalization and also with the "islet of ability" in stimuli discrimination observed in autism.

The question of how a neural circuit with too many or few neurons arises from the process of neurodevelopment was not addressed by the Cohen's model. In the present work, a more realistic model is proposed where, based on the fundamental biological process of neuronal competition, a neural network capable of organizing its structure as a function of the stimuli received will, as in a process of neurodevelopment, recruit more or less neurons and make more or less synapses, bringing insight to the cause of the aberrant neurogenesis of autism. Roughly speaking, this work shows how excessive neuronal inhibition in some neurodevelopmental phase can result in aberrant neural circuits with computational abilities similar to those observed in autism. The computer 
simulations suggest that brain regions responsible for the formation of higher level representations are impaired in autistic patients. The lack of this integrated representation of the world would lead to the peculiar cognitive deficits of socialization, communication, and imagination but could also explain some "islets of abilities" like excellent memory and stimuli discrimination. The neuronal model is based on plausible biological findings and on recently developed cognitive theories of autism.

\section{The Cognitive Theories of Autism}

The generality of the neuropathological findings, allied to the failure of the psychodynamic theories, gave impetus to cognitive scientists in their search for theoretical bridges between autistic brain and behavior.

All the theories of autism are capable of explaining cognitive deficits but none of them touch the question of the "islets of ability" or the spiky profile in the neuropsychological assessment. The recently established weak central coherence theory states that autistic children have a peak of performance in activities that don't require integration but, in contrary, segmentation of information $[4,5]$. In accordance to this theory, autistic brains would have a cognitive style that favors segmentation due to the limitation or impossibility of a more holistic information processing. Experiments on language, memory, and perception [6] have shown that autistic children are not strongly disadvantaged, in relation to normal children, when processing meaningless verbal material. Non-autistic children have a tendency to search for associations and other types of integration of the processed information, having a low performance when unconnected or meaningless material is presented in verbal assessment. Autistic patients also achieve good performance in visuo-spatial tasks where image segmentation is necessary [23]. Findings of less coherence in narratives and superior visuo-spatial skills in non-autistic relatives of autistic children suggest that the lack of central coherence in these patients are genetically transmitted [17]. It is worth noting that adults with autism which can pass the theory of mind tests show cognitive patterns of weak central coherence [8]. This result hints the possibility that weak central coherence is an independent and additional feature of autism, with no obvious relation to the theory of mind deficits, in principle.

\section{Neural Cooperation and Competition}

In the middle of the 19th century the scientists Helmholtz and Mach studied many phenomena of the visual perception in humans. Particularly, they were interested in optical illusions like the fact that edges or contours between light and dark parts of an image tended to be enhanced in relation to the light and dark interior of the image. They explained the illusion hypothesizing that in the human retina the cells are excited by light that converges to a central region and inhibited by the light that projects to the surrounding areas. Almost a century later, experimental results showed that the eye of the crab called Limulus [10] and some vertebrates [16] have an structure, then called oncenter/off-surround, in which a neuron is in cooperation, through excitatory synapses, with the neurons in the immediate neighborhood while it is in competition with the neurons which lay outside these surroundings.

Competition and cooperation are found not only statically hardwired but also as part of many neuronal dynamical processes. As a matter of fact, competition is essential to the neurodevelopment where neurons compete for certain chemicals. In synaptogenesis, for example, the substances generically called neural growth factors are released by stimulated neurons and, spreading through diffusion, reach the neighboring cells, promoting synaptic growth. Cells that receive neural growth factors make synapses and live, while the cells that have no contact with these substances die [12]. A neuron that releases neural growth factor guides the process of synaptic formation in its tridimensional neighborhood, becoming a center of synaptic convergence. When some neighboring neurons release different neural growth factors in different amounts, many synaptic convergence centers are generated and a competition is established between them by the synapses of their surroundings. It is worth noting that, as a single neuron is capable of receiving and releasing neural growth factors at the same time, the two competition processes described above effectively occur in every neuron and, consequently, a signaling network is established to control the development of neural circuits. Remembering that all this competition is started and controlled by environmental stimulation, it is possible to have a glimpse to the way the environment records or represents itself in the brain.

The competition processes described above are essential to the formation of some neuronal organizations called maps. Maps have puzzled neuroscientists in the last decades, mainly the question of how do they arise from the simple on-center/offsurround wiring pattern. Computational theories gave some important insights to the problem, since some cortical maps are artificially developed from simple governing rules of sinaptic plasticity in computer simulation models [18]. The most general of these models is called the Self-Organizing Map [15] in which two sheets of neuronal tissue with $\mathrm{n}$ neurons each, corresponding to the domain and the image, are initially randomly connected in a way that every neuron $\mathbf{i}$ at the image receives synaptic projections $\mathbf{w}_{\mathbf{i}} \in \mathfrak{R}^{\mathbf{n}}$ from every neuron at the domain. Neurons at the domain don't form synapses among themselves and receive "sensory" inputs (stimuli), while neurons at the image make synapses following the on-center/off-surrounding paradigm, i.e., short-range excitation or cooperation and long-range inhibition or competition.

Every time the neural network is in contact with a stimulus $\mathbf{x}_{\mathbf{k}} \in \mathfrak{R}^{\mathbf{n}}, \mathbf{k}=\mathbf{1}, \mathbf{2}, \ldots$ in its domain, there will be 
only one excited neuron $\mathbf{i}^{*}$ at the image. The position $\mathbf{r}^{*}$ of this winner neuron at the image determines how much the synapses will be modified. Synapses from neurons closer to the winner will be strongly changed in such a way that these neurons will be more intensively excited by the stimulus $\mathbf{x}_{\mathbf{k}}$ in a next time. Synapses from neurons distant from the winner will be weakly changed or not changed at all, depending on the dispersion $\sigma$ of the neighborhood function $\phi\left(\mathbf{r i}, \mathbf{r}^{*}\right)$, where $\mathbf{r}_{\mathbf{i}} \in \mathfrak{R}^{\mathbf{n}}$ gives the position of a neuron $\mathbf{i}$ at the image sheet. By this process, every neuron in the image will be more easily excited by the stimulus $\mathbf{x}_{\mathbf{k}}$ (synaptic facilitation) in the future. The development of the map is due to the fact that the amount of synaptic facilitation is proportional to the distance from the winner neuron. The process of synaptic modification $\Delta^{t} \mathbf{w}_{\mathbf{i}}$ for each neuron $\mathbf{i}$ is repeated for every learning step $\mathbf{t}$ where the stimulus $\mathbf{x}_{\mathbf{k}} \in \mathfrak{R}^{\mathbf{n}}, \mathbf{k}=\mathbf{1}, \mathbf{2}, \ldots$ is presented to the neural network, and is given by

$$
\Delta^{\mathrm{t}} \mathrm{w}_{\mathrm{i}}=\rho(\mathrm{t}) \cdot \phi\left(\mathrm{ri}, \mathrm{r}^{*}\right) \cdot\left(\mathrm{x}_{\mathrm{k}}-\mathrm{w}_{\mathrm{i}}\right)
$$

where $\rho(t)$ is the learning rate defined by

$$
\rho(t)=\rho_{0} \cdot \beta^{(t-1)} ; 0<\beta<1, t=1,2, \ldots
$$

The learning rate begins with the value $\rho_{0}$ and decreases with the learning step $\mathbf{t}$ with a rate $\beta$.

The neighborhood symmetric function $\phi\left(\mathrm{r}_{\mathrm{i}}, \mathrm{r}^{*}\right)$ takes the form of a gaussian function like

$$
\phi\left(\mathrm{ri}, \mathrm{r}^{*}\right)=\exp \left(-\left\|\mathrm{r}_{\mathrm{i}}-\mathrm{r}^{*}\right\|^{2} / 2 \sigma(\mathrm{t})^{2}\right)
$$

The initial dispersion of the gaussian, $\sigma_{0}$, is high, representing that all the neurons in the image are considered neighbors. This allows the modification of the randomness of the initial synapses to a more organized pattern where neighborhood is of capital importance. Every time step $\mathbf{t}$ that another stimulus is presented to the neural network domain, the neighborhood shrinks a bit, gradually giving to the map a local organization. The dispersion $\sigma(t)$ at each learning step is given by

$$
\sigma(\mathrm{t})=\sigma_{0} \cdot \alpha^{(\mathrm{t}-1)} ; \quad 0<\alpha<1, \mathrm{t}=1,2, \ldots
$$

where $\alpha$ is a decrement rate.

The way the learning rate decreases and the neighborhood shrinks is fundamental to the map development. A faster decrement in the learning rate does not give enough time to the synapses to change, and so the randomness of the initial synaptic pattern is consolidated at the end of the process. When neighborhoods shrink rapidly, the level of neuronal cooperation necessary to produce maps are not present and neighborhood relationships are ill-defined at the end of the simulation. Indeed, the neighborhood function may be likened to the steady-state concentration profile of a neural growth factor in the neural tissue. When the dynamical equilibrium between neural growth factor release and metabolization is accomplished in every region of the tissue, due to the diffusion process a concentration profile that asymptotically decreases with radial distance is attained. The parameter $\sigma_{0}$ represents the amount of neural growth factor released by the neurons at the beginning of the neurodevelopment process.

An efficient way of assessing the effectiveness of a neurodevelopment process is to measure the total synaptic change occurred. It is observed that large-scale changes in the synaptic pattern get along with successful neural circuits conformation [13]. When synapses are not changed (weakened or strengthened) the neurodevelopment process has not succeeded and the final map is similar to the initial one. At each learning step $\mathbf{t}$ of the self-organizing algorithm, a winner neuron determines the intensity of the synaptic changes for every neuron $\mathbf{i}$ by equation (1). Summing the amount of synaptic change $\Delta^{\mathbf{t}} \mathbf{w}_{\mathbf{i}}$ for all neurons at every learning step $\mathbf{t}$ gives us the total synaptic change $\mathrm{S}$ of the selforganizing process:

$$
\mathrm{S} \equiv \sum_{\mathrm{t}} \sum_{\mathrm{n}}\left\|\Delta^{\mathrm{t}} \mathrm{w}_{\mathrm{i}}\right\|
$$

In the next section, some simulation experiments show how abnormal neural maps may develop from self-organizing processes, resulting in neural networks with computational abilities capable of explaining some cognitive characteristics of autistic children.

\section{Simulation Results}

A self-organizing map to represent the synaptic development between two cortical bidimensional sheets was implemented in the $\mathrm{C}$ computer language. A lower level cortical sheet (here called domain) with 400 neurons receives input stimuli from sensory areas and projects its output to the higher level cortical sheet (here called image) also with 400 neurons. A thousand of input stimuli were randomly generated. The range of the random number generator was changed four times in a way of producing four different sets of 250 stimuli each. Indeed, these sets define clusters of relatively similar input patterns, as can be seen in Figure I, where the stimuli of each cluster are represented over a bidimensional sheet of cortical neurons by the same geometric marker. Simulations performed on a RISC workstation show how ill-developed self-organizing maps have computational characteristics that resemble the phenomenology of the autistic syndromes, mainly the islets of ability.

Initially, the synapses between the domain and the image cortical sheets are defined at random. As a consequence, the stimuli presented to the domain cortical sheet (shown in Figure I) are projected to random positions at the image cortical sheet, as represented in the Figure II. The similarities between the stimuli obviously are not preserved at the higher level cortical sheet, or in other words, no map-like 
representation exists. In all experiments that follow, the domain cortical sheet was excited 25 times with the 4 clusters of 250 stimuli in a random manner. The parameters $\rho_{0}, \alpha$ and $\beta$ are kept constant in $0.8,0.99$, and 0.99 , respectively, for all simulations.

Remembering that $\sigma_{0}$ represents the amount of neural growth factor released by the neurons of the image cortical sheet at the beginning of the neurodevelopment process, in a first simulation this parameter was set to a high value $\left(\sigma_{0}=4.0\right)$, sufficient to the correct development of the map. After the execution of the self-organizing algorithm, the four clusters of stimuli presented to the domain cortical sheet (Figure I) were mapped into four different and welldefined regions, as can be seen in Figure III. During the simulation of the neurodevelopment process, the synapses between the two cortical sheets evolved in time with a total synaptic change $\mathbf{S}$ equal to 11.382 , transforming the initially disorganized map (Figure II) into a clearly organized one (Figure III). In fact, any other stimulus never before presented to the neural network, but similar to one of the 250 stimuli of a cluster, will be mapped directly into the region of the image cortical sheet responsible for the representation of that cluster. Imagining that during one's lifetime a multitude of similar stimuli are presented to one's brain, it is easy to conclude that cortical maps are intelligent structures capable of representing infinitely many instances or variations of a stimulus in a constant, welldefined and small region of the cortex. In other words, neural maps are capable of, based on a few examples or instances, create and hardwire (or represent) a whole category in the cortex. One obvious advantage of this capability is the economy incurred: every stimulus would be represented by one specific neuron, billions of these cells should not be enough for a lifetime.

The construction of these maps is possible just due to the fact that the cortical neurons and their synaptic plasticity governing rules build a neurocomputational circuit capable of extracting from the stimuli their commonalties. The cortical map considers the common features as the principal components (or the central coherence) of the stimulus, while the uncommon characteristics that would hind the process of categorization are disregarded.

A normal brain, charged with well-developed maps, is always, and naturally, searching for the central coherence of every new stimulus that reach the cortex. Finding the central coherence is an extremely important cognitive style of normal minds, since in a complex and ever changing environment it is a great deal to recognize a new situation as similar to an old one for which a good response is already known.

Once analyzed the computational properties and the cognitive implications of well-developed cortical maps, the next two similar simulations will show how illdeveloped maps have characteristics that resemble autistic minds. To achieve this purpose, the parameter $\sigma_{0}$ will be decreased at each simulation, showing how the reduction of neural growth factor degrades the map and changes its computational abilities. Starting with $\sigma_{0}=3.5$ (a reduction of $12.5 \%$ in relation to $\sigma_{0}=4.0$ of the successful map), the self-organizing process departed from the same random-generated map (Figure II) and reached the final state shown in Figure IV. Comparing the two maps (Figure III and Figure IV) we note that the last one was completely reshaped. The regions responsible for the clusters of stimuli assumed different forms and relative dispositions at the image cortical sheet. Nevertheless, the computational, and, consequently, the cognitive, properties of the map are the same: categorization, generalization, central coherence or any other word that express common feature recognition. Having a closer look to this map (Figure IV), however, allows us to observe that some misclassified stimuli are present.

A final and extreme reduction of neural growth factor $\left(\sigma_{0}=1.0\right)$ produces a completely segmented map, as seen in Figure V. This last simulation shows us the "dual map" of the first simulation. Maps with high discriminative capability and weak central coherence extraction (Figure V) represent the other side of the medal in relation to the maps with high generalization property and strong central coherence detection (Figure III). Between these two extremes there are a plethora of self-organizing maps, possibly resulting in different levels of the same neurological syndrome or, worse, nosologically different neurological syndromes with the same basic etiology. The curve depicted in Figure VI shows how the total synaptic change $\mathbf{S}$ varies with the neural growth factor released at the start of the simulated neurodevelopment process. It is possible to conclude that weak central coherence extraction maps are consequence of low levels of neural growth factors. These maps are immature because their synapses could not change (low $\mathbf{S}$ value) and were not submitted to the process of strengthening and consolidation or weakening and elimination [13]. Note that an excessive number of synapses is found in autistic brains [19], and this is in accordance to the low value of the total synaptic change $\mathbf{S}$ found in the weak central coherence maps developed here.

The simulations performed until now allow us to plausibly extrapolate some results to the cognition and symptomatology of autism. To begin with, some authors have noticed that "autistic children do not tend to integrate current experiences with previous impressions" [11] and also that "autistic children miss what is salient and pay attention to what is irrelevant" [7]. These two phrases pop out from our simulations every time a reduction of neural growth factor produces a neural map with less generalization (integration) capability and higher discrimination (attention to details). Highly discriminative cortical maps will lead to a cognitive style where the stimuli are segmented and stored as a function of the details. As a consequence, cognition will be guided by the recognition of parts, possibly promoting a behavior characterized by excessive precision and inflexibility. 
Another cognitive consequence of highly discriminative maps is that information (stimulus) is not categorized but represented by segments or instances. The principle of economy is, then, broken and a huge amount of neurons will be necessary to represent concepts that normally would be mapped in just a small cortical region, as ours simulations have shown. When large cortical regions are responsible for the representation of few detailed concepts, emerges a computational phenomenon called "combinatorial explosion" characterized by inefficient information processing due to insufficient hardware. The low IQ and the restricted interests in just a few subjects remarkably well memorized, indeed - observed in autistic patients may be some results of the combinatorial explosion.

On the other hand, highly discriminative neuronal maps allow the automatic and fast segmentation of information and the representation of details. Possibly, this is the reason why autistic children have islets of ability in memory for raw data (names, phone numbers, calendar, realistic drawing, etc...), efficiently solve puzzles where parts of a context need to be quickly analyzed, and also have good sensory discrimination skills, for example to sounds and music [3].

Remembering that cortical processing is obtained from a series of hierarchical maps, where the input to a higher level map is the output of the immediately lower level map, it is possible to preview that cognitive problems will arise when the first map in the hierarchy classifies similar stimuli in different cortical regions. Perhaps, autistic minds cannot develop language skills because they don't have the proper higher level semantic maps [19] that depend on lower level wellformed maps. Also the social difficulties of the autism would be the result of the impossibility of recognizing emotional and facial expressions, tasks that need the interpretation of contexts and the integration of information that occur in higher level maps.

\section{Conclusions}

Autism is marked by deficits in socialization, communication, and imagination but also by some islets of ability in operative skills involving memory and sensory discrimination. There are many cognitive theories for the disease but only one of them, the weak central coherence theory, is capable of explaining the referred islets of ability. After a detailed analysis of the concept of cortical map, a self-organizing algorithm to simulate the process of neurodevelopment of maps is presented. Some simulations performed showed how maps develop and what are their principal control parameters. One of these parameters was plausibly related to the important chemicals called neural growth factors and simulations proceeded to show that the amount of this substance released during the neurodevelopment process is capable of generating maps with a continuously varying generalization capability. The computational properties of these maps were closely analyzed and related to the cognitive weak central coherence theory of autism. The lack of neural growth factor was shown to produce maps with low generalization property or weak central coherence extraction. Cognitive deficits and, the most important, cognitive skills (islets of ability) observed in autistic children were explained by analogies between the computational properties of the simulated maps and the weak central coherence theory. The analogies allow the authors to propose a neurocomputational model for the autism capable of linking biology to cognition in this disease. Albeit these analogies fit very well as a mean to understand autism from a neurobiological point of view, the existence of ill-developed and highly discriminative cortical maps in autistic brains must be verified experimentally.

\section{Acknowledgments}

We would like to thank to Prof. João Ferreira da Silva Filho, director of the Institute of Psychiatry IPUB/UFRJ and Dr. Marlene Fiszman for all pertinent comments and valuable suggestions. We also thank the support given by the National Research Council (CNPq) and the State Research Foundation (FAPERJ).

\section{References}

[1] Asperger H. D. (1944). Autistischen Psychopathen im Kindesalter. Arch PsychiatNervkrankh, 117: 76-136.

[2] Cohen I. L. (1994). An artificial neural network analogue of learning in autism. Biol Psychiatry, 36: 5-20.

[3] Frith U. (1989). Autism: Explaining the Enigma. Oxford Univ. Press.

[4] Frith U., Happe F. (1994a). Language and communication in the autistic disorders[Review]. Philos Trans R Soc Lond B Biol Sci, 346: 97-104.

[5] Frith U., Happe F. (1994b). Autism: beyond 'theory of mind' [Review]. Cognition, 50: 115-32.

[6] Frith U. (1996). Cognitive explanations of autism. Acta Paediatr Suppl 416: 63-68.

[7] Happe F. (1991). The autobiographical writings of three Asperger syndrome adults: Problems of interpretations and implications for theory. Autism and Asperger Syndrome. Cambridge Univ. Press.

[8] Happe, F. (1994). Wechsler IQ profile and theory of mind in autism: a research note. J. Child Psychol Psychiatry, 35:1461-1471.

[9] Happe F., Frith U. (1996). The neuropsychology of autism. Brain,119: 1377-400.

[10]Hartline, H. K., Ratliff, F. (1957). Inhibitory interactions of receptor units in the eye of Limulus. J. of Gen. Physiol., 40:351-376.

[11]Hermelin B. (1978). Images and Language. Autism: A reappraisal of concept and treatment. Plenum Press, NY.

[12]Kandel E.R. (1991). Cellular mechanisms of learning and the biological basis of individuality. Principles of Neuroscience. Norwalk, Appleton and Lange, 1009-1031.

[13]Kandel E. R., Jessel T. M., (1991). Early experience and the fine tuning of synaptic connections. Principles of Neuroscience. Norwalk, Appleton and Lange, 945- 958.

[14]Kanner L. (1943). Autistic disturbances of affective contact. Nerv. Child, 2: 217-50. 
[15]Kohonen T. (1982). Self-organized formation of topologically correct feature maps. Biol. Cybernetics, 43:59-69.

[16]Kuffler, S. (1953). Discharge pattern and fuctional organization of mammalian retina. J. Neurophysiol, 16:3768.

[17]Land R., Folstein S. E., Isaacs C. (1991). Spontaneous narrative-discourse performance of parents of autistic individuals. J Speech Hear Res., 34: 1339-1345.

[18]Malsburg C. (1973). Self-organization of orientation sensitive cell striate cortex. Kybernetik, 14: 85-100.

[19]Ritter H., Schulten K. (1989). Convergency properties of Kohonen's topology conserving maps: fluctuations, stability and dimension selection. Biol. Cybernetics, 60: 58-71.

[20]Ritvo E., Freeman B. (1978). National Society for Autistic children definition of the syndrome of autism. J Autism Childhood Schizophrenia, 8: 162-169.

[21]Rumelhart D.E., McClelland J. L. (1985). Parallel Distributed Processing. MIT Press, Cambridge, MA.

[22]Rutter M. (1978). Diagnosis and definition of childhood autism. J Autism Childhood Schizophrenia, 8: 139-384.

[23]Shah A., Frith U. (1993). Why do autistic individuals show superior performance on the block design task? J Child Psychol Psychiatry, 34: 1351-64.

[24]Wing L., Gould J. (1979). Severe impairments of social interaction and associated abnormalities in children: epidemiology and classification. J Autism Dev Disord, 9: 11-29.

\section{Figures}

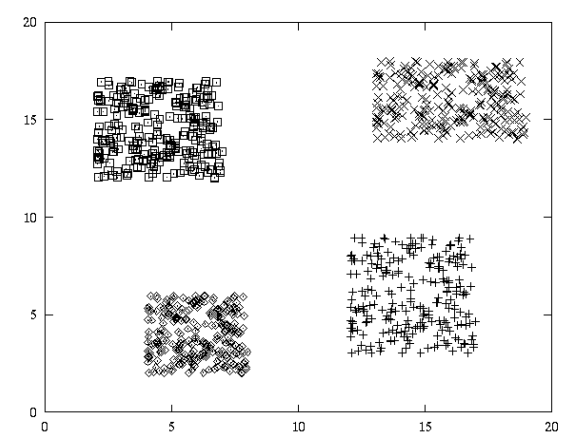

Figure I - A thousand stimuli divided into four clusters of 250 stimuli each.

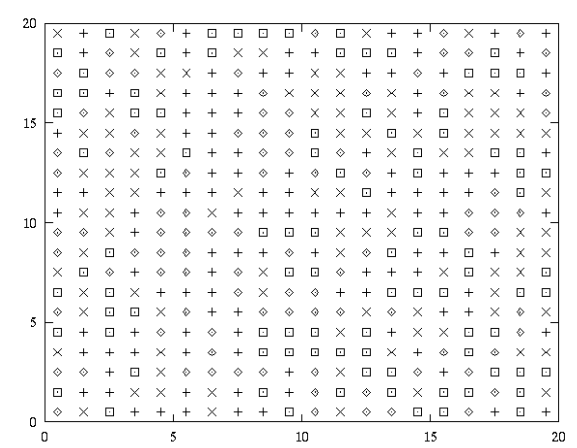

Figure II - Due to the random character of the initial synaptic pattern, the stimuli clusters are projected to the image sheet in a disorganized way.

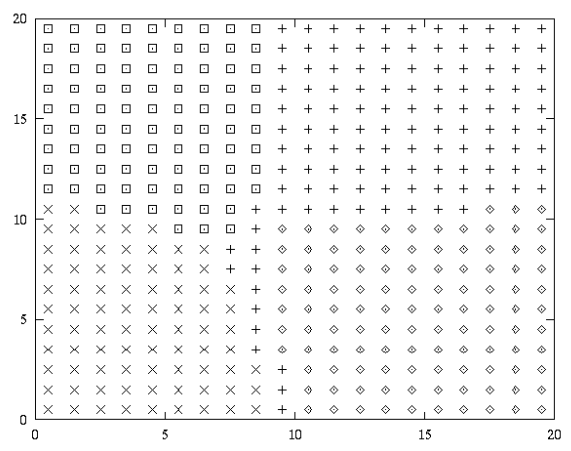

Figure III - A well-developed cortical map generated with sufficient neural growth factor.

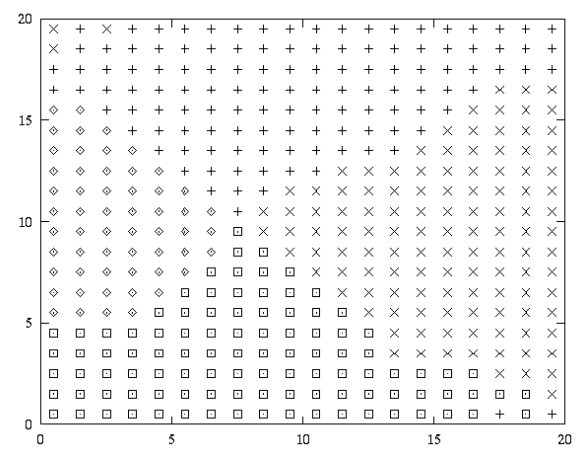

Figure IV - A cortical map developed with a reduced amount of neural growth factor $\left(\sigma_{0}=3.5\right)$. Some stimuli are misclassified.

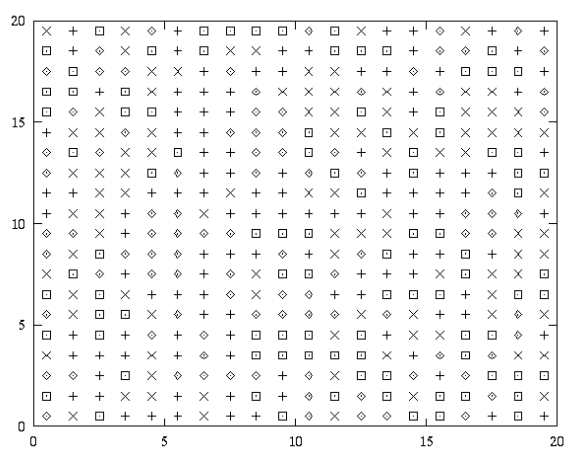

Figure V - An extreme reduction of neural growth factor release $\left(\sigma_{0}=1.0\right)$ generates a completely segmented map.

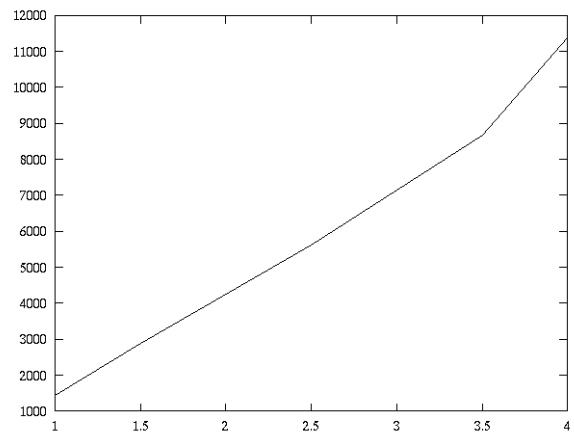

Figure VI - The total synaptic change as a function of the neural growth factor release. 